\title{
FLOW RATIO DESIGN OF PRIMAL AND DUAL NETWORK MODELS OF DISTRIBUTION
}

\author{
G. A. MOHR ${ }^{1}$
}

(Received 27 April, 2000; revised 4 December, 2003)

\begin{abstract}
The finite element method can be used to provide network models of distribution problems. In the present work 'flow ratio design' is applied to such models to obtain approximate minima and maxima for both the primal and dual FEM models. The resulting primal MIN and dual MAX solutions are equal to or close to the exact solutions but, intriguingly, the primal MAX and dual MIN solutions are approximately equal to an intermediate saddle point solution.
\end{abstract}

\section{Introduction}

The distribution problem is one of a number of network problems in management science and operations research $[1,3,4,17]$. Some of these can be usefully viewed as finite element problems [10] and the distribution problem is naturally one of flow in route $i j$ given by

$$
q_{i j}=\left(V_{i}-V_{j}\right) / c_{i j}
$$

where $V_{i}, V_{j}$ are potentials at each end and $c_{i j}$ is the unit transportation cost for this route. Applying (1.1) to the 'truncated' (without slack or other supplementary variables) constraint equations of an optimal distribution network Mohr [11] obtains a result identical to that obtained by summing element matrices

$$
k_{i j}=\frac{1}{c_{i j}}\left[\begin{array}{cc}
1 & -1 \\
-1 & 1
\end{array}\right]
$$

and the resulting network model has the same route flows as in the exact MIN solution (Mohr using a 'direct' LP method for this [11]).

\footnotetext{
'International Arts and Sciences College, 68 Tulip Grove, Cheltenham VIC 3192, Australia.

(C) Australian Mathematical Society 2004, Serial-fee code 1446-181 1/04
} 
Using the finite element method (FEM) to model the initial (with no zero flows) network Mohr [12] uses the steepest descent method to obtain both MIN and MAX solutions close to or equal to the exact solutions (again using the 'direct' LP method to obtain exact MAX solutions [12]).

Then, using $c_{i j}$ in place of $1 / c_{i j}$ in (1.2), dual FEM models are obtained and again applying steepest descent [12], Mohr obtained MIN and MAX solutions close to the exact ones. In the present work a new 'flow ratio design' procedure is applied to these primal and dual FEM distribution models to obtain both MIN and MAX solutions. The method is based on fully stressed design (FSD), a simple but widely used method in finite element analysis of structures in which the solution is iterated, element stresses being calculated at each iteration and used to adjust their structural dimensions according, for example, to the ratio

$$
t_{i+1}=t_{i}\left(\sigma_{i} / \sigma_{\mathrm{lim}}\right),
$$

where $t_{i}$ is the element thickness (the adjusted dimension) in the $i$ th iteration, $\sigma_{i}$ its stress in this iteration and $\sigma_{\text {lim }}$ is the upper stress limit. Then in many problems convergence to an approximately optimal solution is obtained after a few iterations $[2,7,19]$.

Much motivation for FSD as an optimality criterion is given by the classical work of Michell [6] in which it is shown that minimum weight planar truss structures will have constant strain magnitude and take the form of Hencky-Prandtl nets. Subsequently Rozvany and Gollub [16] showed that if the support points for such structures are not fixed in location then the optimum Michell structures consist of straight members.

Using an argument based on generalised constraints with slack variables [9] Mohr also obtains Michell's constant strain condition for optimality, also including a term to allow for vanishing members. The flow ratio method of the present work is, in part, based on this work, except that a 'median' flow value is used in the ratio calculation of (1.3).

\section{Flow ratio design procedure}

In the present work the element constitutive parameters are their unit costs and for minimisation these are adjusted at each iteration using

$$
c_{i j}=R c_{i j}, \quad \text { where } R=\frac{q_{m}}{\left|q_{i j}\right|}, q_{m} \approx \frac{q_{\mathrm{av}}}{2}, q_{\mathrm{av}}=\frac{\sum Q}{N},
$$

where $\sum Q$ is the total flow in the network of $N$ routes and $q_{m}$ is the 'median' flow. For maximisation $c_{i j}=c_{i j} / R$ is used to adjust the element unit costs (iteratively). 
TABLE 1. Results for a $3 \times 3$ problem.

\begin{tabular}{llllllll}
\hline Route & $c_{0}$ & Min & Max & $P_{\min }$ & $D_{\max }$ & $P_{\max }$ & $D_{\min }$ \\
\hline 14 & 5 & 0 & 110 & 0 & 0.01 & 32.50 & 32.50 \\
15 & 10 & 110 & 0 & 110 & 109.98 & 52.50 & 52.50 \\
16 & 10 & 0 & 0 & 0 & 0.01 & 25.00 & 25.00 \\
24 & 20 & $80 / 0$ & $0 / 30$ & 80 & 69.98 & 55.42 & 55.42 \\
25 & 30 & $0 / 80$ & $160 / 130$ & 0 & 90.01 & 75.42 & 75.42 \\
26 & 20 & 80 & 0 & 80 & 0.01 & 29.17 & 29.17 \\
34 & 10 & $60 / 140$ & $30 / 0$ & 60 & 70.01 & 52.08 & 52.08 \\
35 & 20 & $90 / 10$ & $40 / 70$ & 90 & 0.01 & 72.08 & 72.08 \\
36 & 30 & 0 & 80 & 0 & 79.98 & 25.83 & 25.83 \\
\hline$T_{0}$ & & 6700 & 8850 & 6700 & 8297.8 & 7629.2 & 7629.2 \\
$I$ & & & & 12 & 21 & 80 & 150 \\
\hline
\end{tabular}

Then to obtain the minimum solution lower and upper route cost limits

$$
c_{L}=c_{\mathrm{av}} / 40 \rightarrow c_{\mathrm{av}} / 10, \quad c_{U}=10^{6},
$$

where $c_{\mathrm{av}}=\left(\sum c_{i j}\right) / N$, are used.

As already noted, $c_{i j}$ replaces $1 / c_{i j}$ in (1.2) in the dual problem.

For maximisation $c_{L}=0.01$ or 0.001 and $c_{U}=100$ was used in the present work. In later work [14] $c_{L}$ was chosen in the range 10 to $100 \%$ of the value used for minimisation and $c_{U}$ was chosen in the range $5 c_{\mathrm{av}}$ to $100 c_{\mathrm{av}}$, giving almost identical results for the examples studied here.

Observing these limits iteration proceeds and some routes vanish as their $c_{i j} \rightarrow c_{U}$, flows $q_{i j}<0.001$ being set to zero prior to calculating the total distribution cost

$$
T_{0}=\sum\left|q_{i j}\right|\left(c_{i j}\right)_{0}
$$

where $\left(c_{i j}\right)_{0}$ are the initial unit costs for each route.

Note that the lower limit 0.001 for $q_{i j}$ was used with $8 \mathrm{~d}$.p. computation and a value of 0.01 gave the same results and might be needed with less accurate computation.

\section{An example problem}

Table 1 shows the route flow obtained using $q_{m}=25$ for a $3 \times 3$ distribution problem (with 3 supply and 3 demand points) compared to the exact LP solutions (columns 3 and 4$)$. The supply flows $(i=1,2,3)$ are 110,160, 150 and the demand flows $(j=4,5,6)$ are $-140,-200,-80$. 
For minimisation we have $c_{\mathrm{av}}=155 / 9 \approx 17$ and we take $c_{l}=1 \approx c_{\mathrm{av}} / 20$ and $c_{L}$ is in the middle of the range given in (2.1).

Then after $I=12$ iterations (of the primal FEM model) the exact minimum solution (column 5 in Table 1) is obtained, the final element costs being $c_{i j}=c_{L}=1$ for routes with non-zero flows and for the vanishing routes

$$
c_{14}=c_{25}=c_{u}=10^{6}, \quad c_{16}=731629, \quad c_{36}=234271
$$

so that, indeed, in these $q_{i j} \simeq 0$.

For the dual minimum, on the other hand, all the final $c_{i j}=c_{L}=1$ except that $c_{16} \gg 1$ initially but $c_{16} \rightarrow c_{L}$ slowly with iteration (and $c_{16} \simeq 4$ when $I=150$ ). Here an 'intermediate' solution with no zero flows is found, this being the saddle point between the primal and dual solutions.

For the maximum solution the same $q_{m}$ value is used and the cost limits are

$$
c_{L}=0.01 \text { or } 0.001, \quad c_{U}=100 \text {. }
$$

The dual maximum solution (column 6 in Table 1) is only a lower bound to the exact solution (column 4) and the final element costs are $c_{i j}=c_{L}$ for routes with $q_{i j}=0$ and $c_{i j}=c_{U}$ for routes with 'non-zero' flows.

For the primal maximum (column 7 ) the saddle point solution is obtained again, here with all final element $\operatorname{costs} c_{i j}=c_{U}$ except that $c_{16} \simeq 0$ initially but $c_{16} \rightarrow c_{U}$ slowly with iteration (and $c_{16} \simeq 25$ at $I=90$ ).

Note that for this saddle point solution $T_{0}$ is here close to the average of the initial (at $I=1$ ) primal and dual solutions after one iteration, that is,

$$
\left(P_{1}+D_{1}\right) / 2=(7313.5+7929.6) / 2=7621.6 .
$$

Note also that use of a median value for $q_{m}$ here was found by trial to provide satisfactory results, particularly in the case of the primal minimum problem which is that of usual interest. Doubtless improved results for the dual maximum can be obtained with alternative values for $q_{m}$ (and perhaps $c_{U}$ ). Doubtless too the 'dual' appearance of the saddle point solution is the result of use of this median value $q_{m}$, an intriguing result.

\section{Further examples}

Table 2 shows the route flows obtained using $q_{m}=10$ for a $3 \times 4$ problem with supply flows $(i=1,2,3)$ of $60,80,60$ and demand flows $(j=4,5,6,7)$ of -50 , $-40,-70,-40$. Here for minimisation $c_{L}=0.2 \simeq c_{\mathrm{av}} / 15$ was used and the result (column 5) is close to the exact solution. 
TABLE 2. Results for a $3 \times 4$ problem.

\begin{tabular}{llllllll}
\hline Route & $c_{0}$ & Min & Max & $P_{\min }$ & $D_{\max }$ & $P_{\max }$ & $D_{\min }$ \\
\hline 14 & 2 & 50 & 0 & 40 & 0.01 & 15.00 & 15.00 \\
15 & 5 & 0 & 40 & 0 & 0.01 & 11.67 & 11.67 \\
16 & 4 & 0 & 10 & 0 & 59.98 & 21.67 & 21.67 \\
17 & 5 & 10 & 10 & 20 & 0.01 & 11.67 & 11.67 \\
24 & 1 & 0 & 50 & 10 & 0.01 & 20 & 20 \\
25 & 2 & 10 & 0 & 0 & 39.99 & 16.67 & 16.67 \\
26 & 1 & 70 & 0 & 70 & 0.01 & 26.67 & 26.67 \\
27 & 4 & 0 & 30 & 0 & 39.99 & 16.67 & 16.67 \\
34 & 3 & 0 & 0 & 0 & 49.98 & 15.00 & 15.00 \\
35 & 1 & 30 & 0 & 40 & 0.00 & 11.67 & 11.67 \\
36 & 5 & 0 & 60 & 0 & 10.02 & 21.67 & 21.67 \\
37 & 2 & 30 & 0 & 20 & 0.00 & 11.67 & 11.67 \\
\hline$T_{0}$ & & 330 & 760 & 340 & 680.1 & 568.3 & 568.3 \\
$I$ & & & & 16 & 17 & 20 & 18 \\
\hline
\end{tabular}

Once again the 'flow ratio design' (FRD) dual maximum is a lower bound (column 6) of the exact result (column 4-note again the latter is also obtained by the 'direct' LP method [12] but using a dual pivoting rule).

Then $P_{\max }$ and $D_{\min }$ are identical and their $T_{0}$ value is close to the average of $P_{1}$ and $D_{1}\left(P_{1}=464.6\right.$ and $\left.D_{1}=665.1\right)$.

Table 3 shows the route flows obtained using $q_{m}=1.5$ for another $3 \times 4$ problem with supply flows $(i=1,2,3)$ of $7,9,18$ and demand flows $(j=4,5,6,7)$ of $-5,-8,-7,-14$. Here for minimisation $c_{L}=1 \simeq c_{\mathrm{av}} / 40$ was used and the result (column 5) is an upper bound.

The dual maximum $D_{\max }$ is close to the exact solution (column 6) and again $P_{\max }$ and $D_{\min }$ are identical and their $T_{0}$ value is close to the average of $P_{1}$ and $D_{1}\left(P_{1}=1020.4\right.$ and $\left.D_{1}=1543.6\right)$ but still closer to the average of the exact extremal solutions in this instance.

Finally Table 4 shows the route flows obtained using $q_{m}=5$ for a $4 \times 5$ problem with supply flows ( $i=1,2,3,4)$ of $90,75,35,25$ and demand flows $(j=5, \ldots, 9)$ of $-40,-35,-70,-30,-50$. Here demand exceeds supply by 25 units and a dummy supply point 4 with route costs of 50 is introduced to model this situation.

Here for flow ratio minimisation $c_{L}=0.1 \simeq c_{\mathrm{av}} / 40$ was used and the result (column 5) is close to the exact solution. The dual maximum is a lower bound and $P_{\max }$ and $D_{\min }$ are almost identical and their $T_{0}$ values are close to the average of the exact extremal solutions.

Note here that it was found necessary to use $c_{L}=0.5$ (not 0.1 ) to obtain $D_{\min }$ and 
TABLE 3. Results for a $3 \times 4$ problem.

\begin{tabular}{lcrrrlll}
\hline Route & $c_{0}$ & Min & Max & $P_{\min }$ & $D_{\max }$ & $P_{\max }$ & $D_{\min }$ \\
\hline 14 & 19 & 5 & 0 & 0 & 0.00 & 0.50 & 0.50 \\
15 & 30 & 0 & 7 & 0 & 7.00 & 1.50 & 1.50 \\
16 & 50 & 0 & 0 & 0 & 0.00 & 1.50 & 1.50 \\
17 & 10 & 2 & 0 & 7 & 0.00 & 3.50 & 3.50 \\
24 & 70 & 0 & 0 & 0 & 0.00 & 1.50 & 1.50 \\
25 & 30 & 2 & 0 & 2 & 1.00 & 2.00 & 2.00 \\
26 & 40 & 7 & 0 & 7 & 0.00 & 1.50 & 1.50 \\
27 & 60 & 0 & 9 & 0 & 8.00 & 4.00 & 4.00 \\
34 & 40 & 0 & 5 & 5 & 5.00 & 3.00 & 3.00 \\
35 & 8 & 6 & 1 & 6 & 0.00 & 4.50 & 4.50 \\
36 & 70 & 0 & 7 & 0 & 7.00 & 4.00 & 4.00 \\
37 & 20 & 12 & 5 & 7 & 6.00 & 6.50 & 6.50 \\
\hline$T_{0}$ & & 743 & 1548 & 798 & 1530.0 & 1195.5 & 1195.5 \\
$I$ & & & & 19 & 19 & 50 & 50 \\
\hline
\end{tabular}

the same limit was used to obtain $P_{\max }$ (though for the latter the usual value of 0.01 can also be used).

This minor change in procedure was needed because of the identical costs introduced for routes from the dummy supply point 4 , resulting in negative flow for route 48 for $P_{\max }$ and in this route flow cycles between values of 0 and 1 in iteration to obtain $D_{\min }$.

Note too that the initial solution for the dual of this problem results in several negative flows (and consequently $D_{1}=4630.4$ ) and that generally in other problems negative route flows may be introduced, sometimes temporarily, by flow ratio iteration, particularly if $q_{m}$ is not close to $q_{\mathrm{av}} / 2$ when alternative solutions to those found here may be obtained.

\section{Alternative models}

Examples of basis transformation similar to that used to discover a finite element basis for the distribution problem [11] are given by Mohr [8,9]. As an example corresponding to the present FEM distribution model consider a simple spar element with nodes $i, j$ and axial force $F_{i j}$ given by

$$
F_{i j}=E A_{i j}\left(d_{j}-d_{i}\right) / L_{i j}=S_{i j}\left(d_{j}-d_{i}\right),
$$

where $d_{i}, d_{j}$ are the parallel displacements at each node (at the ends) and $L_{i j}, A_{i j}$ are the element length and cross-sectional area. Then here linear interpolation can be 
TABLE 4. Results for a $4 \times 5$ problem.

\begin{tabular}{llllllll}
\hline Route & $c_{0}$ & Min & Max & $P_{\min }$ & $D_{\max }$ & $P_{\max }$ & $D_{\min }$ \\
\hline 15 & 1.5 & 0 & 0 & 30.5 & 0.00 & 16.50 & 16.50 \\
16 & 6.4 & 0 & 35 & 0 & 34.99 & 14.33 & 14.36 \\
17 & 1.8 & 70 & 25 & 45.5 & 21.68 & 24.94 & 24.94 \\
18 & 4.0 & 0 & 30 & 0 & 11.67 & 14.30 & 14.36 \\
19 & 3.5 & 20 & 0 & 14.0 & 21.67 & 19.94 & 19.98 \\
25 & 1.6 & 40 & 0 & 9.5 & 0.00 & 13.50 & 13.50 \\
26 & 2.6 & 35 & 0 & 35.0 & 0.00 & 11.33 & 11.36 \\
27 & 1.9 & 0 & 25 & 24.5 & 28.34 & 21.94 & 21.94 \\
28 & 3.1 & 0 & 0 & 6.0 & 18.33 & 11.30 & 11.36 \\
29 & 5.8 & 0 & 50 & 0 & 28.33 & 16.94 & 16.84 \\
35 & 5.3 & 0 & 35 & 0 & 34.98 & 5.00 & 5.01 \\
36 & 3.5 & 0 & 0 & 0 & 0.01 & 5.00 & 5.02 \\
37 & 2.4 & 0 & 0 & 0 & 0.01 & 12.50 & 12.53 \\
38 & 1.3 & 30 & 0 & 24 & 0.00 & 5.00 & 5.02 \\
39 & 2.2 & 5 & 0 & 11.0 & 0.01 & 7.50 & 7.42 \\
45 & 50.0 & 0 & 5 & 0 & 5.02 & 5.00 & 5.00 \\
46 & 50.0 & 0 & 0 & 0 & 0.00 & 4.35 & 5.00 \\
47 & 50.0 & 0 & 20 & 0 & 19.98 & 10.63 & 10.58 \\
48 & 50.0 & 0 & 0 & 0 & 0.00 & -0.61 & 0.00 \\
49 & 50.0 & 25 & 0 & 25.0 & 0.00 & 5.63 & 5.48 \\
\hline$T_{0}$ & & 1651 & 2162 & 1653.5 & 2095.8 & 1921.8 & 1914.3 \\
$I$ & & & & 10 & 12 & 70 & 30 \\
\hline
\end{tabular}

applied to the parallel displacement $d$ in the element.

If these element forces (in a structure of such elements) were direction independent (in the $x y$-plane) we could sum the element equations (5.1) to form equilibrium equations for each node of the system in the same way as in the distribution problem when its constraint equations are formed (prior to the LP solution). Then for a single element we can write

$$
\left\{\begin{array}{c}
-1 \\
1
\end{array}\right\}\left[F_{i j}\right]=\left\{\begin{array}{c}
F_{i} \\
F_{j}
\end{array}\right\}
$$

and using (5.1) as a basis transformation we obtain

$$
S_{i j}\left\{\begin{array}{c}
-1 \\
1
\end{array}\right\}\left[\begin{array}{ll}
-1 & 1
\end{array}\right]\left\{\begin{array}{l}
d_{i} \\
d_{j}
\end{array}\right\}=\left\{\begin{array}{l}
F_{i} \\
F_{j}
\end{array}\right\}=S_{i j}\left[\begin{array}{cc}
1 & -1 \\
-1 & 1
\end{array}\right]\left\{\begin{array}{l}
d_{i} \\
d_{j}
\end{array}\right\} .
$$

giving the usual element (stiffness) matrix for a 1-D two d.f. element. 
Then if we have an element with an additional central node $k$, we use quadratic interpolation and (5.1) is replaced by [8]:

$$
\left\{\begin{array}{l}
F_{i} \\
F_{j}
\end{array}\right\}=S_{i j}\left[\begin{array}{ccc}
-3 & 4 & -1 \\
1 & -4 & 3
\end{array}\right]\left\{\begin{array}{l}
d_{i} \\
d_{k} \\
d_{j}
\end{array}\right\}=T\{d\} .
$$

Applying linear interpolation for $F_{i j}$ we have the force interpolation

$$
F_{i j}=\left\{\left(1-x / L_{i j}\right),\left(x / L_{i j}\right)\right\}^{t}\left\{F_{i}, F_{j}\right\}=\{f\}^{t}\{F\},
$$

where $\{f\}$ is the vector of (linear) interpolation functions. Then a kernel stiffness matrix is given by

$$
k_{i j}^{*}=S_{i j} \int_{0}^{L_{i j}}\{f\}\{f\}^{t} d x=\frac{S_{i j} L_{i j}}{6}\left[\begin{array}{ll}
2 & 1 \\
1 & 2
\end{array}\right]
$$

and the final element stiffness matrix is given by

$$
k=T^{t} k^{*} T=\frac{E A_{i j}}{6 L_{i j}}\left[\begin{array}{ccc}
14 & -16 & 2 \\
-16 & 32 & -16 \\
2 & -16 & 14
\end{array}\right],
$$

which is the correct result.

Similarly quadratic elements may be transformed to cubic ones and such transformation can also be applied to element mass and geometric stiffness matrices [8,9].

In the distribution problem, however, $q_{i j}$ and $V_{i}, V_{j}$ respectively correspond to $F_{i j}$ and $d_{i}, d_{j}$ in the foregoing. Clearly extension to higher order elements is possible.

As shown by Mohr [11], 2-D continuum FEM models of distribution problems which are similar to network models (and vice versa) are easily formed. The element matrix for a right-angled isosceles triangular element, for example, can be obtained by contraction of the classical Turner triangle by putting Poisson's ratio to zero and superposing the $x$ and $y$ terms, giving [13]

$$
\frac{t}{2 h}\left[\begin{array}{ccc}
2 & -1 & -1 \\
-1 & 1 & 0 \\
-1 & 0 & 1
\end{array}\right]\left\{\begin{array}{l}
\phi_{1} \\
\phi_{2} \\
\phi_{3}
\end{array}\right\}=\left\{\begin{array}{l}
q_{1} \\
q_{2} \\
q_{3}
\end{array}\right\}
$$

where $h$ is the length of the sides at right angles and $t$ the element thickness. This is a useful introduction as it can be formed intuitively using (5.2). This gives some insight into the fundamental resemblance of continuum elements to simple network ones and Mohr [11] compares a network distribution model with a continuum model with 6 node elements, obtaining reasonable similarity.

Then, of course, distribution models with both 2-D and line elements are possible, for example using 2-D elements for a 'background' or general system of minor routes and line elements for main routes. 
TABLE 5. Summary of results.

\begin{tabular}{lllll}
\hline Problem: & 1 & 2 & 3 & 4 \\
\hline Exact: & & & & \\
Min & 6700 & 330 & 743 & 1651 \\
Max & 8850 & 760 & 1548 & 2162 \\
\hline Steepest descent: & & & & \\
Min & 6700 & 340 & 779 & 1651 \\
Max & 8850 & 760 & 1548 & 2160.5 \\
\hline FRD: & & & & \\
Min & 6700 & 340 & 798 & 1653.4 \\
Max: & 8298 & 680 & 1530 & 2095.8 \\
\hline
\end{tabular}

\section{Conclusions}

Table 5 compares extremal solutions for the total $\operatorname{cost} T_{0}$ obtained using the present flow ratio procedure with the exact solutions and those obtained using a steepest descent procedure with 'element access' parameters [12].

As demonstrated by Mohr [9], the steepest descent method can be applied to the optimisation of a wide range of finite element models.

The simple 'flow ratio design' (FRD) approach used in the present work, however, gives good results. Generally we will require only the minimum (primal) solutions in practice and, as the FRD method used here shows, this occurs when all (non-zero) route flows have equal cost.

This is an important result, corresponding to the 'constant strain' character of (optimal) Michell structures [6]. A corresponding constant ' $r / c$ ' ratio result is widely used in cost-benefit analysis. The flow ratio approach used in the present work, therefore, emphasises the wide applicability of such criteria which might, in fact, be viewed as the converse of Pareto's Law in management science [18].

It is shown that such basis transformation, as used to obtain linear (in $V$ ) distribution models, can be used to obtain higher order line elements and that, perhaps in conjunction with these, simple continuum models are also possible.

Finally, note that such optimality criterion methods as FSD or the present FRD method do not guarantee optimal solutions [5]. They are simple and very widely applicable concepts, however, and may often suggest more practical solutions and the FRD method has been successfully applied to traffic flow networks [15].

If the traffic flows are governed by the classical linear flow rule

$$
v_{i j}=V_{i j}\left(1-k_{i j} / K_{i j}\right),
$$

where $v_{i j}$ and $k_{i j}$ are the element traffic velocity and density and $V_{i j}$ and $K_{i j}$ are 
respectively the element free flow velocity and jam density, then the equations for each element are

$$
\left\{\begin{array}{l}
q_{i} \\
q_{j}
\end{array}\right\}=\frac{K_{i j} V_{i j}}{L_{i j}}\left[\begin{array}{cc}
1 & -1 \\
-1 & 1
\end{array}\right]\left\{\begin{array}{l}
P_{i} \\
P_{j}
\end{array}\right\},
$$

where $q_{i}, q_{j}$ are the inflows at each end, $L_{i j}$ is the length of the route and $P_{i}, P_{j}$ are arbitrary potentials at the element nodes.

Setting a datum potential of zero in the network the problem is solved for the nodal potentials $P_{i}$ and the element flows then calculated using

$$
q_{i j}=R_{i j}\left(P_{i}-P_{j}\right), \quad \text { where } \quad R_{i j}=K_{i j} V_{i j} / L_{i j} .
$$

Solving the quadratic equation

$$
q_{i j}=k_{i j} v_{i j}=k_{i j} V_{i j}\left(1-k_{i j} / K_{i j}\right),
$$

two roots $k_{a}$ and $k_{b}$ and their corresponding velocities $v_{a}$ and $v_{b}$ are obtained. In work to date the larger velocity $v_{b}$ is the feasible root.

Both the steepest descent method of Mohr [12] and the present flow ratio design procedure have been successfully used for such traffic flow networks and give the same results for the route flows.

\section{References}

[1] K. M. Bartol and D. C. Martin, Management, 2nd ed. (McGraw-Hill, New York, 1994).

[2] R. H. Gallagher and O. C. Zienkiewicz, Optimum structural design (Wiley, London, 1973).

[3] P. Gilmour, Logistics management: an Australian framework (Longman Cheshire, Melbourne, 1993).

[4] F. S. Hillier and G. J. Lieberman, Introduction to operations research, 3rd ed. (Holden-Day, Oakland CA, 1980).

[5] U. Kirsch, "On singular topologies in optimal structural design", Structural Optimiz. 2 (1990) 133-142.

[6] A. G. M. Michell, "The limits of economy in frame structures", Phil. Mag. Ser. 68 (1904) 589-597.

[7] G. A. Mohr, "Elastic and plastic predictions of slab reinforcement requirements", Civ. Engrg Trans Inst. Engrs Aust. CE21 (1979) 16-20.

[8] G. A. Mohr, "Finite element formulation by nested interpolations: application to a quadrilateral thin plate bending element", Civ. Engng Trans Inst. Engrs Aust. CE25 (1983) 211-218.

[9] G. A. Mohr, Finite elements for solids, fluids, and optimization (Oxford University Press, Oxford, 1992).

[10] G. A. Mohr, "Optimization of critical path models using finite elements", Civ. Engng Trans Inst. Engrs Aust. CE36 (1994) 123-126.

[11] G. A. Mohr, "Finite element modeling of distribution problems", Appl. Math. Comput. 105 (1999) 69-76. 
[12] G. A. Mohr, "Optimization of primal and dual network models of distribution", Comp. Meth. Appl. Mech. Engng 188 (2000) 135-144.

[13] G. A. Mohr, "The patch method: a nodal equation method based on FEM", Adv. Engng Software 32 (2001) 327-335.

[14] G. A. Mohr, Finite elements and optimization for modern management (International Publishers, Melbourne, 2002).

[15] G. A. Mohr, "Finite element modeling and optimization of traffic flow networks", Road and Transport Research (submitted).

[16] G. I. N. Rozvany and W. Gollub, "Michell layouts for various combinations of line supports. Part l", Int. J. Mech. Sci. 32 (1990) 1021-1043.

[17] R. G. Schroeder, Operations management, 3rd ed. (McGraw-Hill, New York, 1989).

[18] C. J. Slaybough, "Pareto's law and modern management", Management Services (March-April 1967).

[19] W. R. Spillers and S. Al-Banna, "Optimization using iterative design techniques", Comput. Struct. 3 (1973) 1263-1270. 